\title{
Temporal variations of directional brightness temperature over a maize canopy in South France
}

\author{
Guoliang TIAN, Tao YU, Yonghong LV \\ LARSIS-IRSA-CAS, Beijing, China \\ e-mail: yutao@irsa.irsa.ac.cn
}

\author{
Roland BOSSENO \\ IRD / CSE-INRA, Avignon, France \\ e-mail: rbosseno@avignon.inra.fr
}

\author{
Xingfa GU, Jean-Francois HANOCQ \\ CSE-INRA, Avignon, France \\ e-mail: gu@avignon.inra.fr
}

\author{
Michel LEGRAND \\ Université des Sciences et Technologies de Lille \\ e-mail: legrand@loa.univ-lille1.fr
}

\begin{abstract}
A field experiment has been conducted from May to August in 1999 over a maize canopy at INRA Avignon branch in France. The experiment covered whole growth period of maize plant in order to observe directional brightness temperature (TBD) variations of row structure canopy as a function of measuring time and date. The TBD was extracted from thermal infrared (TIR) images captured by a TIR camera mounted upon a crane. The results addressed that $T B D$ were highly date and time specific. Comparing the variations of DBT near the noontime with different biomass, the measurements over a middle dense canopy revealed an evident row-direction-oriented hot stripe in DBT polar map, where appeared a hot spot along the sun direction. Similarly, for a low cover condition, a wider hot stripe appeared in the polar map, the hottest area in the band is around solar position. However, for a high biomass condition that the field was nearly whole covered by the leaves, the lowest temperature appeared along the row direction, which formed a cool strip in the polar map. Comparing the variations of DBT on the same day, for a measurement over middle dense canopy, in the morning and afternoon, with a large solar zenith, the range of DBT variation decreased, hot strip features weakened and turned wider, solar position was out of the hot strip. As a conclusion of the field experiment, maize TBD feature is dominated by sun - sensor geometry, complex effects of canopy structures coupled with spatial distributions of canopy brightness temperatures. Row structure effect appeared clearly in TBD polar map throughout the whole measurement. Quantitative explains are expected in the further research on the physical models.
\end{abstract}

Keywords- directional brightness temperature; temporal variation; maize canopy

\section{INTRODUCTION}

Directional brightness temperature (DBT) is one of key indexes characterizing canopy thermal radiation distributions. As a parameter directly captured by remote sensing detectors, DBT has been widely applied in the estimation of agricultural canopy energy budget, the retrieval of field biophysical and phenological parameters, and the directional normalization of remote sensing information obtained from different platforms etc. Many authors have addressed that DBT depends on sun target - sensor geometry, complex effects of canopy structures coupled with various agronomic parameters, and highly date and time specific. [1, 2]. In an air borne experiment, Lagouarde et al. [3] observed the maritime pine stands' directional variation in thermal band and concluded that there is a 'hot spot' structure appear via the viewing direction, the shape of the profile along principal plane is dominant by the age of the trees.

In this paper, we present the observed field DBT variations corresponding to solar direction, plant growth status and soil moisture etc. The objective of this study was to clarify the dominant sources for the variation of angular features of corn field's thermal radiometric radiation, which was helpful for the improvement of DBT models.

\section{MATERIALS AND METHODS}

\section{A. The maize canopy and the equipments for the experiment}

The experimental field locates at INRA-Avignon $\left(43^{\circ} 57^{\prime}\right.$ latitude and $4^{\circ} 5^{\prime}$ longitude) in France. It has a size of 150 meters long in North-South, 100 meters width in West-East direction. The maize was planted north-south oriented on May 10, 1999 (Day of Year: DOY130) with a row distance of $0.8 \mathrm{~m}$ and the plant density is 9.32 plants per $\mathrm{m}^{2}$. The change of leaf area index (LAI) and plant height via date are shown in Table 1 . The experiment lasted nearly three months from the end of May to the Beginning of August.

TABLE I. LAI AND PLANT HEIGHT IN DIFFERENT MEASURING DATE

\begin{tabular}{|c|c|c|c|c|c|c|c|c|}
\hline Date & Jun. 4 & Jun. 16 & Jun. 24 & Jul. 2 & Jul. 9 & Jul. 20 & Jul. 30 & Aug. 6 \\
\hline DOY & 155 & 167 & 175 & 183 & 190 & 201 & 211 & 218 \\
\hline LAI & 0.2 & 0.75 & 1.73 & 3.07 & 3.89 & 4.6 & 4.8 & 4.7 \\
\hline Height(m) & 0.3 & 0.5 & 0.8 & 1.2 & 1.5 & 1.7 & 1.85 & 1.99 \\
\hline
\end{tabular}

The in situ experiment was conducted by a camera-crane system, which consists of one wide band TIR camera, a sixband visible-near infrared camera, an industrial crane and a multidirectional platform. The platform is mounted on the crane bar, which is 20 meter over the ground. The TIR camera

This study is supported by INRA France and China's Funds (Grant No. 2000077902, 2002AA130010-2, and CX020011) 
employed in the experiment was INFRAMETRICS Model 760. It equipped with an $80^{\circ}$ wide angle lens. The special window of the camera is $7.25 \mu \mathrm{m}$ to $13.25 \mu \mathrm{m}$.

\section{B. The measurement and data process}

In the measurement, the camera viewed to the nadir firstly, and then recorded the TIR information. After the footprint viewing, camera directed to at least four directions continuously with $45^{\circ}$ inclination to receive field directional thermal radiance in the measurement, each viewing lasted about 20 seconds. The sampling rate kept constant of one image per second. So there were about 20 images for one direction viewing, their average brightness temperature is used as true value to minimize the high frequency fluctuation effect and improve the measurement accuracy. Each off nadir viewing image has a common area with nadir viewing image. In the common area of off viewing and nadir viewing images, pixels of two images have the same direction, the difference of their average value is used to indicate field temporal changes due to the difference of measuring time.

By using a TIR camera, measuring and sampling could be divided into two steps in the acquisition of target's DBT. In the field measurement, the approach is designed to shorten the measurement period in account of temporal errors. For the same reason, sampling process for extracting field directional feature from the measurement has also been optimized. Generally, sampling depends on the target's geometric structure, field directional radiation features, environment changes, and the geometry of measurement. Various methods for sampling design have been proposed for different purposes [4]. Sampling design concerns sample size selection and sampling interval selection. We design our sample size in this way that: sample of $3 \mathrm{~L}$ dimension for nadir viewing $\left(\theta=0^{\circ}\right)$, so that FOV effect limits within 3\%; sample of $5 \mathrm{~L}$ dimension for near nadir viewing $\left(2^{\circ}<\theta<20^{\circ}\right)$, FOV effect limits within $3 \%$; sample of $10^{\circ}$ FOV for the off nadir measurement $(\theta$ $>20^{\circ}$ ), FOV effect limits within $3 \%$, sample size increase with the viewing inclination. In large zenith condition, whether the sample contains an integer number of rows turns to be unimportant because a large number of rows are in the viewing scene. In the selection of sampling interval, strip structure method was used: the images were divided into several strips vertical to the row direction, the wide of the strips corresponds to sample's sizes. So the stripes' interval in row direction is of sample's size dimension. Within the strips, samples distance is $1 / 3$ length of sample size. During the sampling process, some of pixels are used twice for calculation which leads a smooth effect for the curves. In the data processing, some flaws in the images, such as the route in the field, the shadow of crane and sensor, metal structures in the field, sampling area damaged for geo parameters measurement need to be eliminated. Generally, there still left a difference in common place of images after eliminating the temporal effect in the combination process, this bias is unavoidable which caused two temperature values at the same direction in the polar map. We used the average temperature as the value. At last, a smoothing express process of 'cubic' interpolation was performed to fill the area without data for forming the final DBT presentation.
Polar coordinate presentation is used to express maize DBT. North is designed as the original direction whose azimuth is $0^{\circ}$. In clockwise, the angles for east, south and west are $90^{\circ}$, $180^{\circ}$ and $270^{\circ}$ respectively. In local midi time, the solar azimuth is $180^{\circ}$.

\section{RESULTS AND DISCUSSION}

\section{A. Measurement at different time on the same day}

Figure 1 shows temporal changes of maize canopy DBT on June 24, 1999 (DOY 175), the profiles in solar principal plane and perpendicular plane are also presented. During the day, canopy's height was $0.8 \mathrm{~m}$, LAI is 1.73 ; average wind speed of 20 minutes at the height of one meter over canopy was $0.8 \mathrm{~m} / \mathrm{s}$, maximum instant value was $2.6 \mathrm{~m} / \mathrm{s}$, minimum value was smaller than that of the detector's sensitivity; the field was irrigated two days before, the surface of soil was in drought condition, the soil moisture at 5 centimeter depth was 0.20 $\mathrm{cm}^{3} / \mathrm{cm}^{3}$. Table 2 shows solar positions in 6 DBT measurements. $\theta_{\mathrm{s}}$ and $\varphi_{\mathrm{s}}$ are solar zenith and azimuth respectively.

TABLE II. DBT MEASUREMENT TIME ON JUNE 24, 1999

\begin{tabular}{ccccccc}
\hline Local time & $7: 56$ & $8: 29$ & $13: 10$ & $13: 55$ & $15: 33$ & $16: 02$ \\
\hline$\theta_{\mathrm{s}}\left({ }^{\circ}\right)$ & 52 & 46 & 26 & 33 & 49 & 54 \\
$\varphi_{\mathrm{s}}\left(^{\circ}\right)$ & 93 & 99 & 222 & 241 & 264 & 269 \\
\hline
\end{tabular}

In all the measurements, hot areas appear in solar radiation direction, while the ranges of angular variations change greatly. In the morning at eight o'clock of local time, DBT's range is only $2^{\circ} \mathrm{C}$, the profile's fluctuation is $1^{\circ} \mathrm{C}$. Viewing from the sun's direction which is vertical to the row direction, the field was nearly whole covered because of large viewing zenith. Vegetation received the solar radiation directly and was warmed up. The soil part obscured by the vegetation still kept its cool status, their temperature difference was very small. So the heterogeneity of field thermal radiation was presented clearly and no asymmetry structure of principal line and perpendicular line appeared. One hour later, the field component temperatures hadn't an obviously change, the brightness temperature was only $2^{\circ} \mathrm{C}$, large fluctuation was still the main feature of the profile lines. In these two measurements, the brightness temperature of nadir view is very low. In the early afternoon, field brightness temperature reached to its peak of the day. At the time, part of soil heated by the solar radiation directly had the highest temperature value of the field, its temperature rises quickly which is great faster than that of corn canopy and shaded soil, and played a dominant role in the angular viewing variations. At 16 o'clock of local time, the sun ran to right west vertical to the row direction, with a large solar zenith, the soil was obscured by the corn again which lead to a quick redraw of soil brightness temperature and decrease of the field average temperature, the DBT range decreased to $3^{\circ} \mathrm{C}$, large fluctuation appeared again. In all the measurements conducted in the afternoon, hot band appears in the polar maps. Row structure plays an important 
role in the forming of field DBT. For the observations at different time in the same day, the changes of DBT largely depend on component brightness temperature changes within the scene. The importance of solar position had been discussed by many authors [1-5]. The sun position decides the ratio between sunlit soil and shaded soil. Its move caused redistribution of field component fractions within the scenes. Besides, the solar position has a strong relation with field component brightness temperature value, which also depends on some other key parameters of the field.
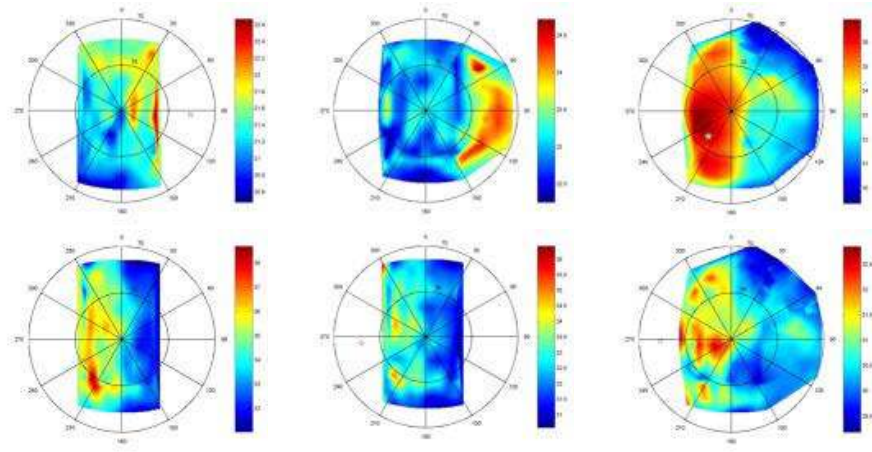

Figure 1. Temporal changes of maize canopy DBT on June 24, 1999 (DOY 175)

\section{B. Measuring at the similar time in different date}

Figure 2 shows three plots of field DBT and their variations in solar principal and perpendicular planes. The measurements were conducted in the early afternoon on different days from June 16 to July 20. Together with the forth map (d) in Figure 1, there are four observations at similar time in different growing period. In these observations, wind speed maintains within a low level that the maximum average value was less than $2.1 \mathrm{~m} / \mathrm{s}$, maximum instant value was less than $4.9 \mathrm{~m} / \mathrm{s}$, the sky was in clear condition, the influence of cloud was nibble and was neglected. Table 3 shows the parameters of DBT measurements on early afternoon during the vegetation growth period.

TABLE III. THE PARAMETERS OF DBT MEASUREMENTS

\begin{tabular}{|c|c|c|c|c|}
\hline Date/DOY & Local time & $\theta \mathrm{s}\left(^{\circ}\right)$ & $\varphi s\left(^{\circ}\right)$ & Soil moisture $(\mathrm{m} 3 / \mathrm{m} 3)$ \\
\hline June $16 / 167$ & $13: 20$ & 28 & 229 & 0.14 \\
\hline June $24 / 175$ & $13: 55$ & 33 & 241 & 0.20 \\
\hline July 9/190 & $14: 23$ & 36 & 145 & 0.12 \\
\hline July 20/201 & $14: 50$ & 42 & 251 & 0.18 \\
\hline
\end{tabular}

The hot area appear in all the maps, however, the shapes of hot area and profile lines change with the increase of biomass amount. A wide band shape hot area is displayed on the polar map of June 16 corresponding to a low cover fraction and low corn height. The band is along row direction, hottest area in the band is around solar position. During this measurement, most part of soil was shined directly. Dried soil was heated quickly and reached to a temperature much higher than vegetation. Canopy had small fraction in the scenes of nadir and small zenith viewing, which lead the hot band to be very wide. For large zenith viewing, canopy fraction increases rapidly, which make field surface temperature decrease quickly, its limitation is the canopy's temperature. Within the whole viewing range, the temperature difference reached up to $8^{\circ} \mathrm{C}$, which is the maximum value observed in these three maps. Figure 2 (c) displays DBT variation for higher biomass condition, the field was nearly whole covered by the corn leaves. Contrary to low biomass DBT, the lowest temperature appeared in the nadir viewing that there is a cool band appear in the polar map. In this measurement, soil part's brightness temperature is lower than of leaves' in the afternoon of local time. Under near whole cover condition, less solar radiation could penetrated the vegetation layer and reach the soil surface, especially when the radiation direction is no along the row direction. Within a long period, the soil under the canopy couldn't be heated by direct solar radiation. Besides, with the increase of cover and LAI, we hypothesize microclimatic environment also plays an important role due to the heat transfer, evapotraspirations, multi scattering of radiation within the canopy, field brightness temperature distributions is smoothed by these heat exchanges. So the temperature differences among field components are dramatically decrease. In this map, the temperature variation of whole angular range is less than $1.5^{\circ} \mathrm{C}$.
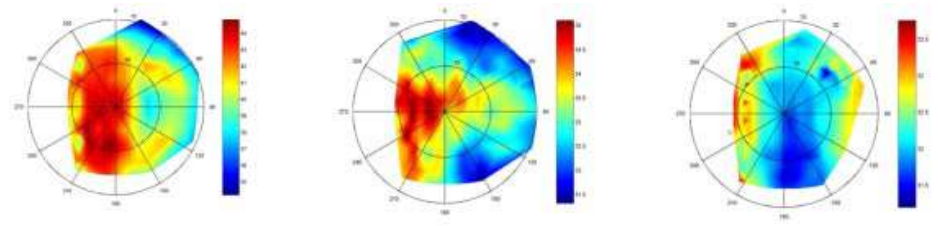

Figure 2. DBT measurements at the same time during the vegetation growth

\section{CONCLUSION}

Experimental study of the corn field DBT allows classifying the reasons for its variations and supports the analysis of their influences. The results obtained over different dates and times in different natural conditions reveal angular effects exist in most of the growth period and measuring time. Its range and shape are various according to measuring date and time. Corn geo structure and solar position have a dominant effect on the final result. Soil moisture and wind speed also play an important role in the forming of DBT due to its influence on the soil background thermal characteristics and energy exchanges within the field. Quantitative explains are expected in the further research.

\section{REFERENCES}

[1] W. P. Kustas, "Ground and aircraft infrared observations over a partially-vegetated area”. International journal of remote sensing, 11: 409-427, , 1990.

[2] L. Prévot, "Modélisation des echanges radiatifs au sein des couverts végétaux. Application a la télédétection. Validation sur un couvert de maïs, Doctoral Thesis, University of Paris VI,’, 1985.

[3] L.P. Lagouarde, H. Ballans., P. Moreau, D. Guyon and D. Coraboeuf, "Experimental study of brightness surface temperature angular variations of maritime pine(pinus pinaster) stands". Remote sensing of environment, 72: 17-34, 2000.

[4] S. R. Sandmeier, "Acquisition of bidirectional reflectance factor data with field goniometers", Remote sensing of environment, 73, 247-269, 2000.

[5] W. P. Kustas, "Ground and aircraft infrared observations over a partially-vegetated area," Int. J. Remote Sens., vol. 11, pp. 409-427, 1990. 